\title{
MINIMAL NONNILPOTENT SOLVABLE LIE ALGEBRAS
}

\section{ERNEST L. STITZINGER}

Abstract. We shall say that a solvable Lie algebra $L$ is a minimal nonnilpotent Lie algebra if $L$ is nonnilpotent but all proper subalgebras of $L$ are nilpotent. It is shown here that if $L$ is a minimal nonnilpotent Lie algebra, then $L$ is the vector space direct sum of $A$ and $F$ where $A$ is an ideal in $L, F$ is a one-dimensional subalgebra of $L$, either $A$ is a minimal ideal of $L$ or the center of $A$ coincides with the derived algebra, $A^{\prime}$, of $A$ and in either case $F$ acts irreducibly on $A / A^{\prime}$.

P. Hall and G. Higman have shown in [5] that a nonnilpotent finite group $G$ all of whose proper subgroups are nilpotent can be considered as the product of subgroups $P$ and $Q$ where $P$ is cyclic of prime power order, $p^{\alpha}, Q$ is an invariant $q$-subgroup of $G, q \neq p, \phi(P) \leqq Z(G)$ and $Q$ is either elementary abelian or $\phi(Q)=Z(Q)=[Q, Q]$ where in either case $P$ acts irreducibly on $Q / \phi(Q)$. We shall find a result on solvable Lie algebras which is roughly analogous to this result.

Let $L$ be a finite-dimensional solvable Lie algebra and let $M$ be a self-normalizing maximal subalgebra of $L$. The maximal ideal of $L$ contained in $M$ will be called the core of $M$. The intersection of all maximal subalgebras of $L$ will be denoted by $\phi(L)$ and is an ideal in $L$ by Lemma 3.4 of [2]. The derived algebra of $L$ will be denoted by $L^{\prime}$ and the center of $L$ by $Z(L)$. We first show the following result, the group theory analogue of which is shown in [4].

Proposition. Let $L$ be a solvable Lie algebra and let $H$ be a selfnormalizing maximal subalgebra of $L$. Let $K$ be the core of $H$. Then

(1) $L / K$ contains a unique minimal ideal $A / K$,

(2) $L / K$ is the semidirect sum of $A / K$ and $H / K$,

(3) $\phi(L / K)=0$, and

(4) $L / K$ is not nilpotent.

Proof. Let $\bar{H}=H / K, \bar{L}=L / K$ and $\bar{A}$ be a minimal ideal of $\bar{L}$. Since $\bar{A}$ is abelian and $\bar{H}$ is maximal in $\bar{L}, \bar{A} \cap \bar{H}$ is an ideal in $\bar{L}$ contained in $\bar{H}$. Since $\bar{H}$ contains no nonzero ideals of $\bar{L}, \bar{A} \cap \bar{H}=0$. Then, since $\bar{H}$ is maximal in $\bar{L}, \bar{A}+\bar{H}=\bar{L}$. If $\bar{B}$ is another minimal ideal of $\bar{L}$, then the centralizer of $\bar{A}$ in $\bar{L}, C_{\bar{L}}(\bar{A})$ properly contains $\bar{A}$. Then $\bar{H} \cap C_{\bar{L}}(\bar{A})$ is an ideal in $\bar{L}$ contained in $\bar{H}$, hence $\bar{H} \cap C_{\bar{L}}(\bar{A})=0$ and,

Received by the editors April 6, 1970 and, in revised form, April 17, 1970.

AMS 1970 subject classifications. Primary 17B30.

Key words and phrases. Fitting decomposition, Engel's Theorem. 
therefore, $\bar{A}$ is unique. Since $\bar{H}$ is maximal in $\bar{L}, \phi(\bar{L}) \subseteq \bar{H}$. But $\phi(\bar{L})$ is an ideal in $\bar{L}$, hence $\phi(\bar{L})=0$. Finally, since $\bar{H}$ is a self-normalizing maximal subalgebra of $\bar{L}, \bar{L}$ is not nilpotent by Proposition 3, p. 56 of $[3]$.

THEOREM 1. Let L be a nonnilpotent, solvable Lie algebra all of whose proper subalgebras are nilpotent. Then $L$ is the vector space direct sum of $A$ and $F$ where $A$ is an ideal of $L, F$ is a one-dimensional subalgebra of $L$ and either $A$ is a minimal ideal of $L$ or $A^{\prime}=Z(A)$. In either case, $F$ acts irreducibly on $A / A^{\prime}$.

Proof. By the theorem of [1], $L$ contains a self-normalizing maximal subalgebra $H$ and $H$ is clearly a Cartan subalgebra of $L$. Let $K$ be the core of $H$ in $L$. By the Proposition, $L / K$ contains a unique minimal ideal $A / K$ which complements $H / K$ in $L / K$. Then $L / A$ $\simeq H / K$ and, since $H$ is nilpotent, $L / A$ is nilpotent. Since $L / K$ is not nilpotent and $A / K$ is abelian, using Engel's Theorem, there exists $x \in L / K, x \notin A / K$ such that ad $x$ is not nilpotent. Since $H / K$ is nilpotent and $H / K$ complements $A / K$ in $L / K$, ad $x$ restricted to $A / K$ is not nilpotent. Then the subalgebra $B / K$ of $L / K$ generated by $A / K$ and $x$ is not nilpotent and since $A / K$ is an ideal of $L / K$, $\operatorname{dim} B / K=1+\operatorname{dim} A / K$. Hence $B / K=L / K$ and $\operatorname{dim} H / K=\operatorname{dim} L / A$ $=1$. Hence there exists a one-dimensional subalgebra $F$ of $L$ which is a complement of $K$ in $H$ and is also a complement of $A$ in $L$.

Let $L=H+L_{1}$ be the Fitting decomposition of $L$ with respect to $H$. Then $H / K$ is a Cartan subalgebra of $L / K$ and $L_{1}+K / K$ is the Fitting one-component of $L / K$ with respect to $H / K$. Since $L / K$ $=A / K+H / K, A / K$ is a minimal ideal of $L / K$ and $L / K$ is not nilpotent, $H / K$ acts nontrivially and irreducibly on $A / K$. Since $\operatorname{dim} H / K=1$ and $H / K$ is a Cartan subalgebra of $L / K, A / K$ is the Fitting one-component of $L / K$ with respect to $H / K$. Hence $L_{1}+K / K$ $=A / K$ and $L_{1}+K=A$. Furthermore, since $\left[K, L_{1}\right] \subseteq\left[H, L_{1}\right] \subseteq L_{1}$, $\left[K, L_{1}\right] \subseteq K \cap L_{1}=0$.

Let $T$ be the subalgebra of $L$ generated by $L_{1}$. Since $L_{1}$ is $F$-invariant, $T$ is also, and since $L_{1} \subseteq A, T \subseteq A$. Now $F+T$ is a nonnilpotent subalgebra of $L$, hence $F+T=L$. Then, since $T$ is $F$-invariant, $A=T$. If $K=0, L_{1}=A$ is a minimal ideal of $L$. Suppose then that $K \neq 0$. We claim that $K$ is abelian. Since $A$ is generated by $L_{1}$, we need only consider elements of $K$ of the form $[x, y], x, y \in L_{1}$. Using the Jacobi identity on elements of this type and that $\left[K, L_{1}\right]=0$, one sees that $[K, K]=0$. Then $[A, K] \subseteq[K, K]+\left[L_{1}, K\right]=0$ and $K \subseteq Z(A)$. If $K \subset Z(A)$, then, since $Z(A)$ is an ideal in $L, Z(A)$ is $F$-invariant and hence $Z(A) \cap L_{1}$ is $F$-invariant. But then $Z(A)=A$ which forces 
$K=0$, a contradiction. Hence $Z(A)=K$. Since $A / K$ is abelian, $A^{\prime} \subseteq K$. But $L_{1}$ is a set of generators for $A$, therefore $K \subseteq A^{\prime}$ and $A^{\prime}=K$. Finally, since $F$ acts irreducibly on $A / K$, the proof is complete.

The proof of Theorem 1 indicates we can state this result in the following way.

CoROllaRy. Let $L$ be a nonnilpotent, solvable Lie algebra all of whose proper subalgebras are nilpotent. Let $H$ be a self-normalizing maximal subalgebra of $L, K$ be the core of $H$ and $L=H+L_{1}$ be the Fitting decomposition of $L$ with respect to $H$. Then $L$ is the vector space direct sum of $K, L_{1}$ and $F$ where $F$ is a one-dimensional subalgebra of $L$ and $K+F$ $=H$. Furthermore $A=K+L_{1}$ is an ideal of $L$ and either $K=0$ and $L_{1}$ is a minimal ideal of $L$ or $A^{\prime}=Z(A)=K$. In either case, $F$ acts irreducibly and nontrivially on $A / A^{\prime}$.

\section{REFERENCES}

1. D. W. Barnes, Nilpotency of Lie algebras, Math. Z. 79 (1962), 237-238. MR 27 \#180.

2. D. W. Barnes and H. M. Gastineau-Hills, On the theory of soluble Lie algebras, Math. Z. 106 (1968), 343-354. MR 38 \#1130.

3. N. Bourbaki, Livre XXVI: Groupes et algêbres de Lie. Chap. 1, Actualités Sci. Indust., no. 1285, Hermann, Paris, 1960. MR 24 \#A2641.

4. P. Hall, On system normalizers of a soluble group, Proc. London Math. Soc. (2) 43 (1937), 507-528.

5. P. Hall and G. Higman, $O$ n the p-length of p-soluble groups and reduction theorems for Burnside's problem, Proc. London Math. Soc. (3) 6 (1956), 1-42. MR 17, 344.

North Carolina State University, Raleigh, North Carolina 27607 